\title{
LDPC-Coded OFDM Transmission Based on Adaptive Power Weights in Cognitive Radio Systems
}

\author{
Seyed Eman Mahmoodi, Bahman Abolhassani \\ School of Electrical Engineering, Iran University of Science and Technology (IUST), Tehran, Iran \\ E-mail:e_mahmoodi@elec.iust.ac.ir,abolhassani@iust.ac.ir \\ Received October 9, 2011; revised November 17, 2011; accepted November 30, 2011
}

\begin{abstract}
In this paper, we propose a new scheme to improve the performance of an LDPC-coded OFDM based cognitive radio (CR) link by applying adaptive power weights. To minimize estimation errors of detected signals in all the CR subcarriers, power weights are allocated to the CR subcarriers at the secondary transmitter. Some constraints for the power weights are considered, such as keeping the interference power introduced by the CR to primary users below a given interference threshold and also keeping sum of transmission powers in all CR subcarriers within a total transmission power. The LDPC decoder applies these power weights in the Log Likelihood Ratios (LLRs) used in message passing scheme at the secondary receiver to achieve more reliable communications. So, the received signal in each CR subcarrier will be decoded with the knowledge of transmission power weights, which come from the cognitive feedback channel without additional cost. Simulation results demonstrate that our proposed scheme achieves a lower bit error rate and a higher transmission rate compared with those of the same scheme without applying power weights.
\end{abstract}

Keywords: Cognitive Radio, OFDM, LDPC, Interference, Power Weighting

\section{Introduction}

Transmission of data over wireless communication systems grows rapidly. Cognitive Radio (CR) systems have made it feasible to utilize the frequency spectrum, dynamically and efficiently. A CR system allows coexisting of an unlicensed secondary user (SU) with a licensed primary user (PU) on the same frequency spectrum. However, CR systems should consider the mutual interference introduced by these two groups of users to each other. If this mutual interference is not considered or is considered inaccurately, the spectrum efficiency of both systems could be corrupted. So, one of the main goals for a cognitive radio is to provide a reliable besides high data rate transmission in coexistence with a PU [1].

On the other hand, Low Density Parity Check (LDPC)coded Orthogonal Frequency Division Multiplexing (OFDM) systems have many advantages, which provide both reliability and high data rate communications for CR systems. OFDM transmission makes it feasible to null the subcarriers whose frequency bandwidths are occupied by PUs. As well, applying FFT in this modulation provides an ease of implementation for analysis of the spectrum in other tasks of CR [2]. Moreover, LDPC codes can improve bit error rate (BER), considerably in fading channels by applying appropriate decoders [3].

So far, performances analysis of LDPC-coded OFDM systems have been discussed in different communication environments [3-9]. In [3], the authors study the BER of LDPC codes in block fading channels. They consider that channel state information (CSI) is known in both transmitter and receiver. As well, the use of iterative message passing technique is considered as an efficient approach and is used for decoding in these error correction codes [4]. The utilization of Log Likelihood Ratio (LLR) during this iterative decoding as the reliability factor is investigated and it is shown that the BER is improved [5]. Moreover, use of message passing scheme for decoding of LDPC codes is considered jointly with cooperative spectrum sensing, which is discussed in [6]. Applying LDPC codes in a CR is considered in [7], too. The authors in [7] show that how much improvement in BER can be made by different LDPC decoding algorithms.

Throughput maximization of an LDPC-coded OFDM system is also very important, specifically for high data rate wireless transmission [8]. In [8], the authors derive the optimal power allocation for an LDPC coded system 
to improve the throughput of the system. However, the problem of mutual interference introduced by different systems to each other is a high priority constraint for throughput maximization in CR systems. In [9], the authors analyze the performances of LDPC-coded OFDM systems while users are experiencing the mutual interference. However, in this paper, we consider these systems in cognitive environment. In [10], effect of noise plus interference power introduced by PUs to the CR system is investigated, and the interference power introduced by SUs to the Pus is considered as a constraint. However, this paper does not consider throughput maximization of the CR system. In our paper, we propose an iterative scheme for message passing in the LDPC decoder, while in the secondary transmitter, power weights for each OFDM subcarrier is considered to improve both BER and throughput of the CR. Moreover, since there is a cognitive feedback channel between secondary transmitter and secondary receiver, we apply these power weights in the LDPC decoder as used in the secondary transmitter. This causes improvement in the performance of the CR for error corrections.

The rest of this paper is organized as follows. Section 2 describes the system model. In Section 3, we propose our new scheme in two subsections. First, we achieve the power weights and then apply LDPC coding by these power weights. Section 4 discusses simulation results by comparing with classical scheme. Finally, Section 5 concludes the paper.

\section{System Description}

We consider a single link between a secondary transmitter and a secondary receiver while there are many PUs, which have occupied $M$ OFDM subchannels. This single CR communication link uses LDPC-coded OFDM whose block diagram is shown in Figure 1.

As it can be observed from Figure 1, there is a cognitive feedback channel in CR systems, which provides the information related to spectrum sensing, estimation of interference threshold and channel state information (CSI) to the secondary transmitter [1]. This information is provided by secondary receiver or cognitive sensors, which usually sense the spectrum cooperatively and then inform the secondary transmitter by a fusion center. In addition to dynamic spectrum decision, the secondary transmitter in the system model applies this information for power allocation. We consider that this feedback channel is perfect (no error in received information).

Using the Shannon capacity formula for the CR system, and considering an ideal coding scheme, the transmission rate at the OFDM subcarrier $i$ is given by:

$$
R_{i}\left(P_{i}, h_{i}^{(s s)}\right)=\Delta f \log _{2}\left(1+\frac{\left|h_{i}^{(s s)}\right|^{2} P_{i}}{\sigma_{w}^{2}+\sum_{m=1}^{M} J_{i}^{(m)}}\right),
$$

where the frequency spacing between CR subcarriers is $\Delta f \mathrm{~Hz}, \sigma_{w}^{2}$ denotes the variance of thermal noise and $P_{i}$ denotes the transmission power of the CR subcarrier $i$. As well, $\left|h_{i}^{(s s)}\right|^{2}$ is the instantaneous channel gain of the subcarrier $i$ between SU's transmitter and receiver and $J_{i}^{(m)}$ shows the interference power introduced by PU's subchannel $\mathrm{m}$ to the CR subcarrier $i$. There are M subchannels which are occupied by PUs. Since, the CR system has no information about instantaneous channel gains between PUs' transmitters and the secondary re-

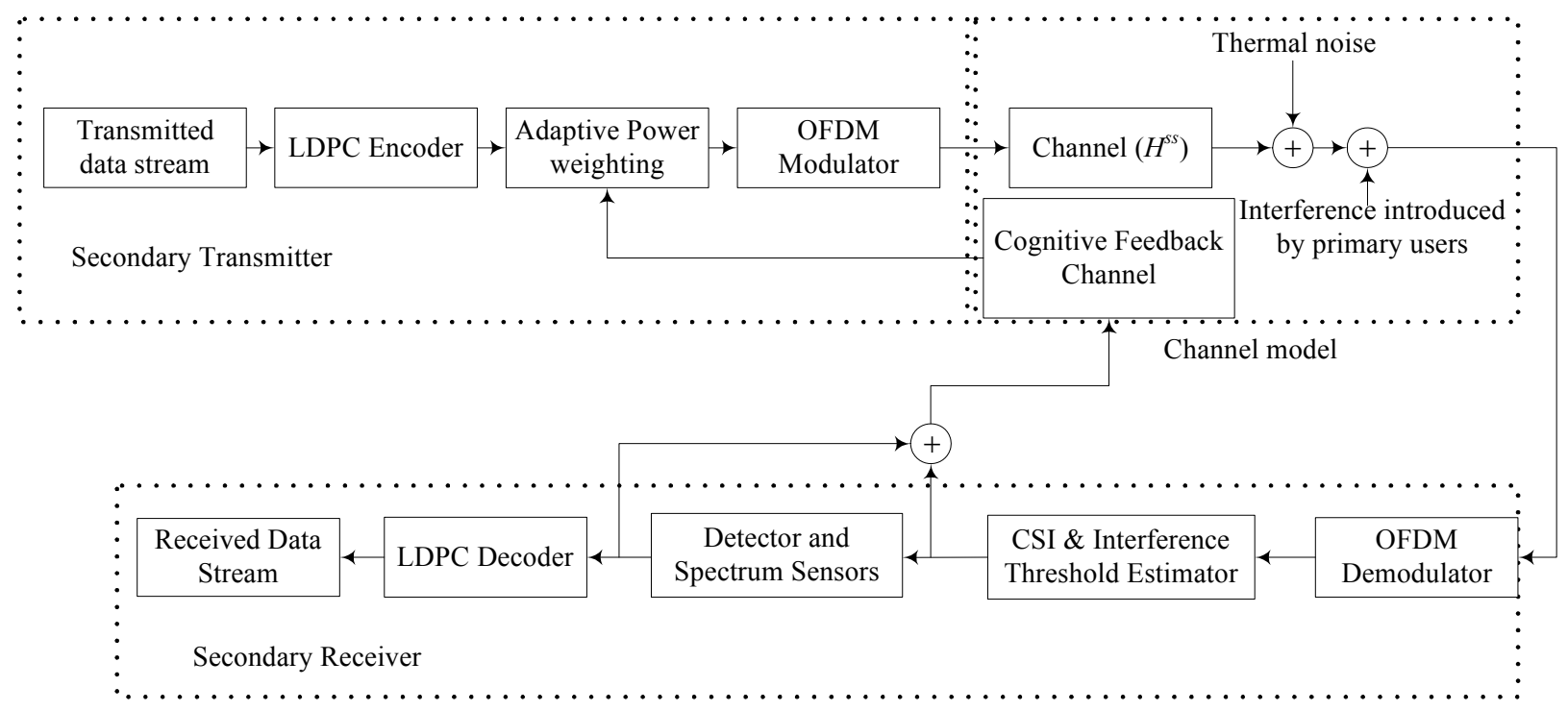

Figure 1. Block diagram of LDPC-coded OFDM in a cognitive radio link. 
ceiver and modulation strategy of PUs, precise calculation of the interference power introduced by PUs is infeasible for the CR. On the other hand, estimation of these interference powers, $J_{i}^{(m)}$ (for all $m=1,2, \cdots, M$ ), is possible at the SU's transmitter. However, for considering PUs' activities, the CR needs to update the estimation of the interference powers, periodically. This is impractical for large number of OFDM subchannels, which consists of CR subcarriers and the subchannels occupied by PUs. This makes the CR very slow to perform estimation at the secondary receiver and then feedback to the SU's transmitter for transmission power weighting.

Since, we consider an imperfect spectrum sensing and we cannot estimate noise variance plus the sum interfereence power introduced by PUs accurately, applying LDPC coding seems an efficient solution to have a CR system with an acceptable performance. To make this LDPC coding even more efficient, using message passing scheme, power weights are passed to the LDPC decoder to be used for a more efficient decoding.

We assume a linear model for the received voltage signal, as

$$
y(i)=w_{i} h^{(s s)}(i) x(i)+n(i),
$$

where $w_{i}$ denotes the signal weight of CR subcarrier $i$. Also, $x(i)$ and $y(i)$ are the transmitted and received voltage signals in CR subcarrier $i$, respectively and $n(i)$ denotes total power of interference generated by $\mathrm{M}$ subchannels of PUs and received by the CR receiver in subcarrier $i$ and thermal noise, which is AWGN with zero mean and variance $\sigma_{w}^{2}$. The receiver should estimate $\hat{x}(i)$, the transmitted signal in each subcarrier. Using [11], the LS estimation of $\hat{x}(i)$ is given by

$$
\hat{x}(i)=\frac{y(i)}{w_{i} \sqrt{P_{0, i}} h^{(s s)}(i)}=x(i)+\frac{n(i)}{w_{i} \sqrt{P_{0, i}} h^{(s s)}(i)},
$$

where $P_{0, i}$ denotes the initial power of CR subcarrier $i$, which has equal value for all $N$ subcarriers, that is

$$
P_{0, i}=\frac{P_{T}}{N} \quad \forall i=1,2, \cdots, N
$$

In Equation (4), noise and the interference coming from PUs are included in $w_{i}$. Also, from Equation (4), the variance of the LS estimation of $\hat{x}(i)$ is given by

$$
\Delta_{i}=\frac{1}{w_{i}^{2} P_{0, i}} \frac{\sigma_{w}^{2}+\sum_{m=1}^{M} J_{i}^{(m)}}{\left|h^{(s s)}(i)\right|^{2}} .
$$

This equation shows that to minimize $\Delta_{i}$, for small values of the ratio of the channel gain to noise plus interference, $w_{i}^{2}$ weight of transmission power should be increased.

\section{Proposed Scheme}

In this section, we propose our new scheme which consists of two important phases. In the first phase, we achieve transmission power weights in all CR subcarriers at the secondary transmitter. Then, using these power weights in the LDPC decoder of the CR receiver, we improve system performance of the $\mathrm{CR}$ in the second phase. In the following two subsections, we explain these two phases.

\subsection{Phase I: Calculations of Power Weights}

Knowledge on the summation of interference powers introduced by PUs is infeasible in this system model, and applying Equation (1) for rate maximization in LDPCcoded system is inefficient. So, we consider the objective function proposed in [12], to achieve power weights. We write the summation of estimation errors of $\hat{x}(i)$, $i=1,2, \cdots, N$, for all $\mathrm{N}$ subcarriers of the CR system as

$$
\Delta=\Delta\left(w_{1}, w_{2}, \cdots, w_{N}\right)=\sum_{i=0}^{N-1} \Delta_{i}=\sum_{i=0}^{N-1} \frac{\sigma_{w}^{2}+\sum_{m=1}^{M} J_{i}^{(m)}}{w_{i}^{2} P_{0, i}\left|h^{(s s)}(i)\right|^{2}} .
$$

By considering Equation (7) normalized to the noise variance plus summation of interference powers introduced by PUs as the objective function, we solve optimization problem to allocate adaptive transmission power weights for each secondary subcarrier. Then, we use these weights in the LDPC decoding.

By minimizing $\Delta$, signal estimation errors decrease. In other words, by maximizing inverse of $\Delta$, actually, we consider the maximization of the total signal to interfereence plus noise (SINR) in all CR subcarriers. However, as was expressed in system description, the CR is unaware of the transmission power levels of the PUs and their channel gains to the secondary receivers, so calculation of the interference introduced by PUs is infeasible. So, we normalize the sum of estimation errors $(\Delta)$ to noise plus sum interference. This normalization results in calculation of suboptimum transmission power weights $\left(w_{i}^{2} s\right)$. However, the optimization problem would be feasible without knowing the noise plus interference power. Also, the optimization problem has some constraints, which should be noted for reliable communications in the primary system and total transmission power of the SU. This optimization problem could be mathematically written as,

$$
\max _{w_{i}} \sum_{i=0}^{N-1} w_{i}^{2} P_{0, i}\left|h^{(s s)}(i)\right|^{2}
$$


subject to,

$$
\begin{gathered}
\sum_{m=1}^{M} \sum_{i=0}^{N} I_{i}^{(m)}\left(d_{i, m}, w_{i}^{2}\right) \leq I^{(t h)}, \\
\sum_{i=0}^{N} w_{i}^{2} P_{0, i} \leq P_{T},
\end{gathered}
$$

where $I_{i}^{(m)}\left(d_{i, m}, w_{i}^{2}\right)$ represents the interference power introduced by CR subcarrier $i$ to the primary subchannel $m$, which is a function of power weight in that subcarrier and the spectral distance between the CR subcarrier $i$ and primary subchannel $m$, written by $d_{i, m}$ [13]. By normalizing Equation (7) to noise plus sum interference and inversing $1 /\left(w_{i}^{2} P_{0, i}\left|h^{(s s)}(i)\right|^{2}\right), \forall k=1,2, \cdots, N$, we maximize total received signal powers in all CR subcarriers using Equation (8), instead of minimizing Equation (7). It's notable that in this optimization problem the interference powers introduced to the PUs should be kept below an interference threshold, $I^{(t h)}$ (Inequality (9)). Also, Inequality (10) represents that the sum value of the weighted transmission powers in $N$ secondary subcarriers should be $P_{T}$, at maximum. Although, the objective function in Equation (8) causes increasing the transmission power, both Inequalities (9) and (10) prevent increasing transmission powers to meet threshold interference $\left(I^{(t h)}\right)$ and peak transmit power $\left(P_{T}\right)$.

By applying convex optimization for this problem, defining the cost function by Equation (8), applying Lagrange multipliers $(\alpha$ and $\beta$ ) for two constraints in Inequalities (9) and (10) and considering Karush-Kahn-Tucker (KKT) Conditions [14], we have

$$
\frac{-1}{P_{0, i}\left|h^{(s s)}(i)\right|^{2}}+\left[\left(\alpha \sum_{m=1}^{M} I_{i}^{(m)}\right)+\beta P_{0, i}\right] w_{i}^{4}=0 .
$$

From Equation (11), $w_{i}^{2}$ can be derived as

$$
w_{i}^{2}=\sqrt{\frac{1}{\left[\left(\alpha \sum_{m=1}^{M} I_{i}^{(m)}\right)+\beta P_{0, k}\right]\left[P_{0, i}\left|h^{(s s)}(i)\right|^{2}\right]}} .
$$

To obtain $\alpha$ and $\beta$, we use a similar algorithm proposed in [12]. We consider the two constraints expressed in Inequalities (9) and (10). By setting power weights obtained using Equation (12) into Inequalities (9) and (10) and applying an iterative algorithm, which is mentioned below in two steps, these parameters would be obtained.

1) With respect to the definition of $\beta$ for the constraint of total power, we first set $\beta=0$ in Equation (12) to obtain $\alpha$ by Inequality (9), as

$$
\alpha=\frac{P_{T}}{N}\left(\frac{1}{I^{(t h)}} \sum_{i=1}^{N} \frac{\sqrt{\sum_{m=1}^{M} I_{i}^{(m)}}}{\left|h^{(s s)}(i)\right|}\right)^{2} .
$$

2) Using $\alpha$ obtained from Equation (13) and assuming initial $\beta$, we check the total power constraint given by Inequality (10). Iteratively, we add to previous $\beta$ until the total power constraint in (10) is satisfied.

Since by applying Step 2, all the power weights decrease, so the interference constraint given by Inequality (9) would not be violated in iterations. Therefore, by obtaining the two Lagrange multipliers, power weights are obtained. Moreover, we observe that these power weight are independent from noise plus sum interference.

\subsection{Phase II: LDPC Decoding Using Power Weights}

In the first phase, we used power weights of OFDM signals in CR subcarriers at the secondary transmitter to reduce the estimation errors of detected signals. Use of error correction codes, by applying Low-Density Parity Check (LDPC) codes, which is one of the most efficient coding schemes in wireless communications, is investigated in the second phase by applying the power weights obtained in Phase I. Since, we have applied a blind scheme to transmit signals without knowledge on the interference power of PUs to each CR subcarrier; use of wireless channel coding schemes, such as LDPC codes improves efficiency of the CR system against the noise plus interference. So far, we have assumed ideal coding in our power loading schemes. However, by encountering unsteady noise plus interference power, use of LDPC codes in the CR system could be considered for error correction to recover the variance of noise plus interfereence uncertainty. It's notable that by applying LDPC codes, transmission rate and error rate of the CR system is improved against interference and noise. In the LDPC decoder, we use message passing scheme to correct bit errors as an iterative solution. The power weights obtained in Phase I of the proposed algorithm and Equation (12) could be considered in the Log Likelihood Ratio (LLR) of iterative message passing scheme in the LDPC decoder to reduce error rates or increase the transmission rate of the CR system. So, the error rate performance of the LDPC coded OFDM system with an iterative decoder is totally characterized without knowledge on the interference power of PUs on the CR receiver.

We assume a sparse parity check matrix with coding rate of $1 / 2$. As shown in [5], the log-likelihood ratio (LLR) with BPSK modulation is given by 


$$
\begin{aligned}
& L_{n}=\ln \frac{\operatorname{pr}\left(s_{n}=1 \mid y_{n}^{(\mathrm{dem})}\right)}{\operatorname{pr}\left(s_{n}=-1 \mid y_{n}^{(\mathrm{dem})}\right)}=\frac{2 y_{n}^{(\mathrm{dem})}}{\sigma^{2}}, \\
& n=1,2, \cdots, K,
\end{aligned}
$$

where $s_{n}$ and $y_{n}^{(\mathrm{dem})}$ are $n$ 'th transmitted and received bit streams among $K$ bits in each packet and $\sigma^{2}$ denotes the AWGN noise plus interference power.

Considering the power weights obtained by Equation (12) and the iterative algorithm mentioned in 3.1, we set the LLRs based on these power weights. Since, we have no knowledge about interference powers in CR subcarrier $i$; we consider noise variance, $\sigma_{w}^{2}$, instead of noise plus interference. So, for the variance of transmitted signals in CR subcarrier $i$, we write

$$
\tilde{\Delta}_{i}=\frac{\sigma_{w}^{2}}{w_{i}^{2} P_{0, i}\left|h^{(s s)}(i)\right|^{2}}, i=1,2, \cdots, N .
$$

Theorem: the LLR in CR subcarrier $i$ for $n$ 'th bit stream by received signal $y_{n, i}^{(d e m)}$ can be set as

$$
L_{n, i}=\frac{2 w_{i}^{2} y_{n, i}^{(\mathrm{dem})}}{\sigma_{w}^{2}}, n=1,2, \cdots, K, i=1,2, \cdots, N .
$$

which causes to improve the error rate performance in the new scheme.

proof: The conditional probability density function (pdf) of received bit stream $n$ in this system model can be considered as

$$
p_{y_{n, i}}=\frac{1}{\sqrt{2 \pi \sigma^{2}}} \exp \left(-\frac{\left(y_{n, i}-\sqrt{P_{0, i}} w_{i} s_{n}\right)^{2}}{2 \sigma^{2}}\right) .
$$

By assuming uniform distribution of probability in BPSK modulation $\left(\operatorname{pr}\left(s_{n}=1\right)=\operatorname{pr}\left(s_{n}=-1\right)=0.5\right)$, and considering (14), obtained LLR from the channel is written as

$$
\begin{aligned}
& L_{n, i}=\ln \frac{\operatorname{pr}\left(s_{n}=1 \mid y_{n, i}^{(\mathrm{dem})}\right)}{\operatorname{pr}\left(s_{n}=-1 \mid y_{n, i}^{(\mathrm{dem})}\right)}=\frac{2 w w_{i}^{2} P_{0, i} y_{n, i}^{(\mathrm{dem})}}{\sigma^{2}}, \\
& n=1,2, \cdots, K, i=1,2, \cdots, N,
\end{aligned}
$$

On the other hand, we should consider the value of noise plus the interference power introduced by PUs to the CR $\left(\sigma^{2}\right)$. Since, we have no information to estimate the interference power introduced by PUs; this could be estimated as AWGN variance [15]. So, we have

$$
\sigma_{w}^{2}+\sum_{m=1}^{M} J_{i}^{(m)} \simeq \sigma^{2}
$$

where $\sigma^{2}$ is the variance of normal distribution. So, by considering the normalized variance of transmitted signals in CR subcarrier $i$ (Equation (15)), the variance of
LLR could be written based on $\sigma_{w}^{2}$. Therefore Equation (16) is concluded from Equation (18). It's notable that the achieved LLR is normalized to the fix value of $P_{0, i}$.

We observe that the LLR given by Equation (16) relies on demodulated signal bits, noise variance and the transmission power weights in each CR subcarrier, which is a function of the channel gain between SU's transmitter and SU's receiver, and interference introduced to the PUs by CR subcarriers. Therefore, by comparing the received signals decoded by the usual message passing scheme with the one decoded by the new scheme (applying weighted LLRs in the decoder), better error rate performance for the new scheme is achieved.

Thus, we have an adaptive weighted LDPC-coded OFDM system. By this channel coding in the CR system, we use the power weights and derive the proposed scheme to reduce the error rate of the LDPC-coded CR system based on OFDM. It is notable that in this coded system model; transmission rate in unit of bits/subchannel can be given by [9]

$$
R=\frac{N \times I_{s}}{B},
$$

where $N$ denotes the number of CR subcarriers, Is represents number of information bits per subcarrier, and $\mathrm{B}$ denotes number of subcarrier bits per OFDM symbol.

In summary, we illustrate the two phase proposed scheme in a flowchart, which is shown in Figure 2. In the $\mathrm{CR}$, we initialize transmit power of CR subcarriers with uniform distribution. From the cognitive feedback channel, we have the CSI between secondary transmitter and secondary receiver and sum interference power, which is introduced by CR to the PUs. Due to power weights calculations, we use a loop. By initialization of Lagrange multiplier $\beta$ and satisfying Constraint (9) in the optimization problem, we obtain Lagrange multiplier $\alpha$. So, we calculate power weights by Equation (12). Now, if these power weights satisfy Constraint (10), desired power weights are achieved. Else, by adding appropriate $\varepsilon$ to $\beta$, power weights are decreased to finally satisfy Constraint (10). So, power weights for all CR subcarriers are achieved and used for CR transmission. We apply these power weights in LLRs of the LDPC decoder for each bit stream for all CR subcarriers to improve the system performance. As we illustrated in this section and we observe in the next section, by applying Equation (16) instead of Equation (14) in message passing, error rate decreases and transmission rate of the CR system increases.

\section{Simulation Results}

In this section, BER and transmission rate of the pro- 


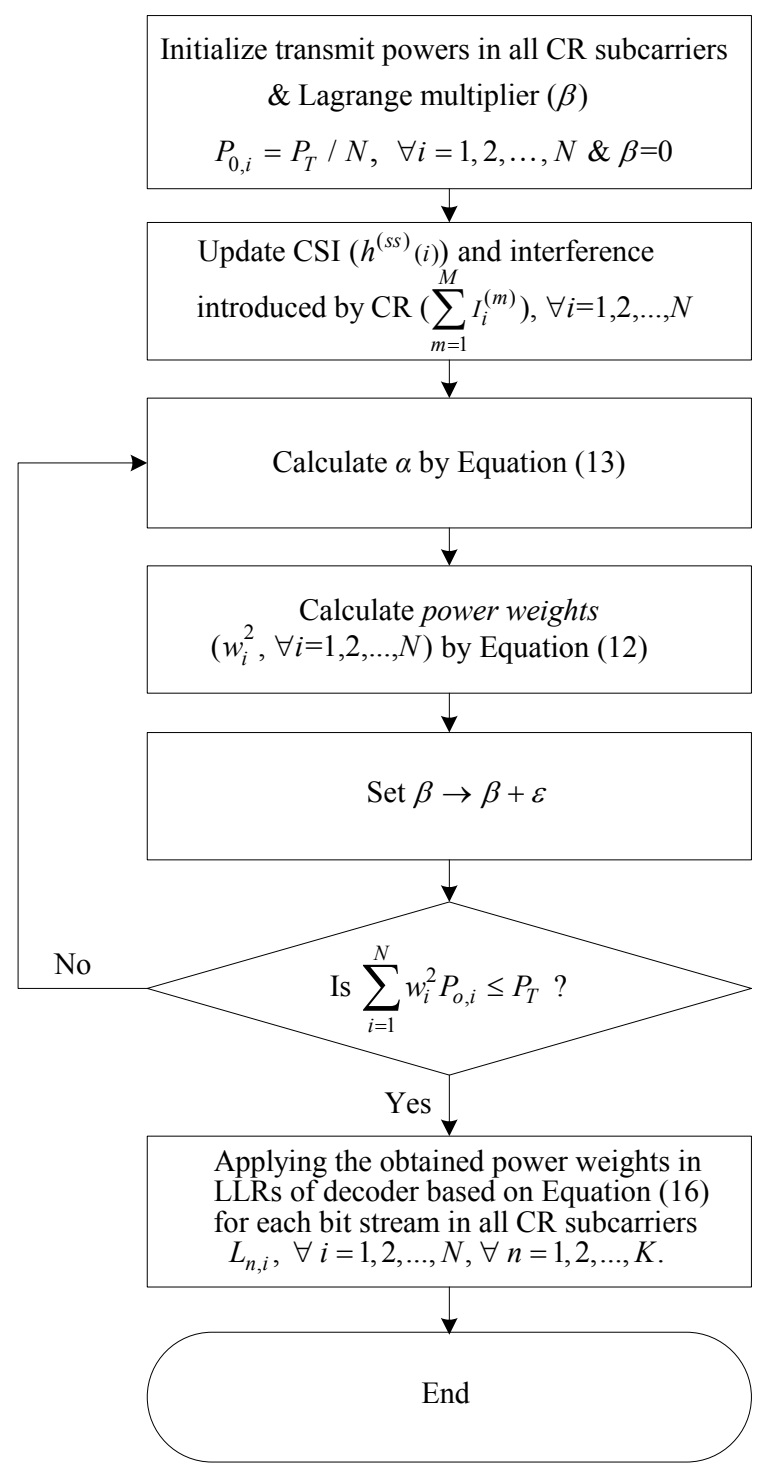

Figure 2. Flowchart of the proposed scheme.

posed two phase scheme is evaluated by using simulation results. Here, we consider the system model, which is discussed in Section 2 by simulation parameters as shown in Table 1. We consider a weighted LDPC coded-OFDM based CR system coexisting with a number of PUs with $M$ occupied subchannels. SU's receiver senses spectrum and feedbacks data to the SU's transmitter. In simulations, we consider a uniform distribution for spectral situations of OFDM subchannels which have been occupied by PUs. Then, by considering the value of false alarm probability and detection of occupied subchannels, spectrum sensing is done. By knowing the CSI between secondary transmitter and receiver, and the sum interference introduced to PUs by CR, power weighting is performed. According to the flowchart, which is shown in Figure 2, the power weights are obtained and sent to
Table 1. Simulation parameters.

\begin{tabular}{cc}
\hline Parameter & Value \\
\hline \# of CR subcarriers $(N)$ & 40 \\
\# of occupied PUs' subchannels $(M)$ & 24 \\
False alarm probability & 0.15 \\
Symbol duration $\left(\mathrm{T}_{\mathrm{s}}\right)$ & $0.4 \mu \mathrm{s}$. \\
Coding rate & $1 / 2$ \\
PU's transmit power in each occupied subchannel & $2 \times 10^{-4} \mathrm{~W}$ \\
\hline
\end{tabular}

the decoder. In the second phase, LDPC decoding is performed by applying the obtained power weights in the LLRs, which is obtained and proved in the previous section. By using these new LLRs in each demodulated bit stream, and comparing the received bit stream to the transmitted bit stream, error rate can be calculated. As well, transmission rate is presented in terms of total transmission power and the interference power introduced by CR to the PUs. In these simulations $N$ which is the number of subcarriers allocated to the $\mathrm{CR}$ and $M$ are 40 and 24 , respectively. We assume that $30 \%$ of PUs occupies the specified frequency bandwidth of the primary system. False alarm probability is 0.15 . In the CR system, sampling time is $0.4 \mu \mathrm{s} ; 5$ bits are used in each frame and 20000 frames are applied for each packet. As well, we consider a $20 \times 10$ parity check matrix made by using sparse LU decomposition method [16]. Channel model between SU's transmitter and receiver, and channel model between SU's transmitter and each of PUs' receivers are assumed to be Rayleigh fading and average channel gain of these channels is $-10 \mathrm{~dB}$.

We consider our proposed scheme, which applies message passing for the LDPC decoding with the LLRs relied on the power weights of the transmitted signal in each CR subcarrier and we compare its performance with the same LDPC coded-OFDM based CR system without applying power weights in the message passing scheme for decoding.

Figure 3 shows bit error rate (BER) of the proposed scheme versus received SINR for our proposed scheme and the same scheme without using power weights, both experiencing CR environment. In this figure, the interference threshold introduced to the PUs is assumed to be $2 \times 10^{-4} \mathrm{~W}$. We observe that our proposed scheme achieves lower error rates by increasing SINR, so that BER of our proposed scheme is $4 \times 10^{-4}$ at SINR $=10 \mathrm{~dB}$, while BER of the same system without using power weights increases to $5 \times 10^{-4}$, which is more than ten times higher.

In Figure 4, we plot the transmission rate of the CR system in Megabits/seconds versus total transmission 


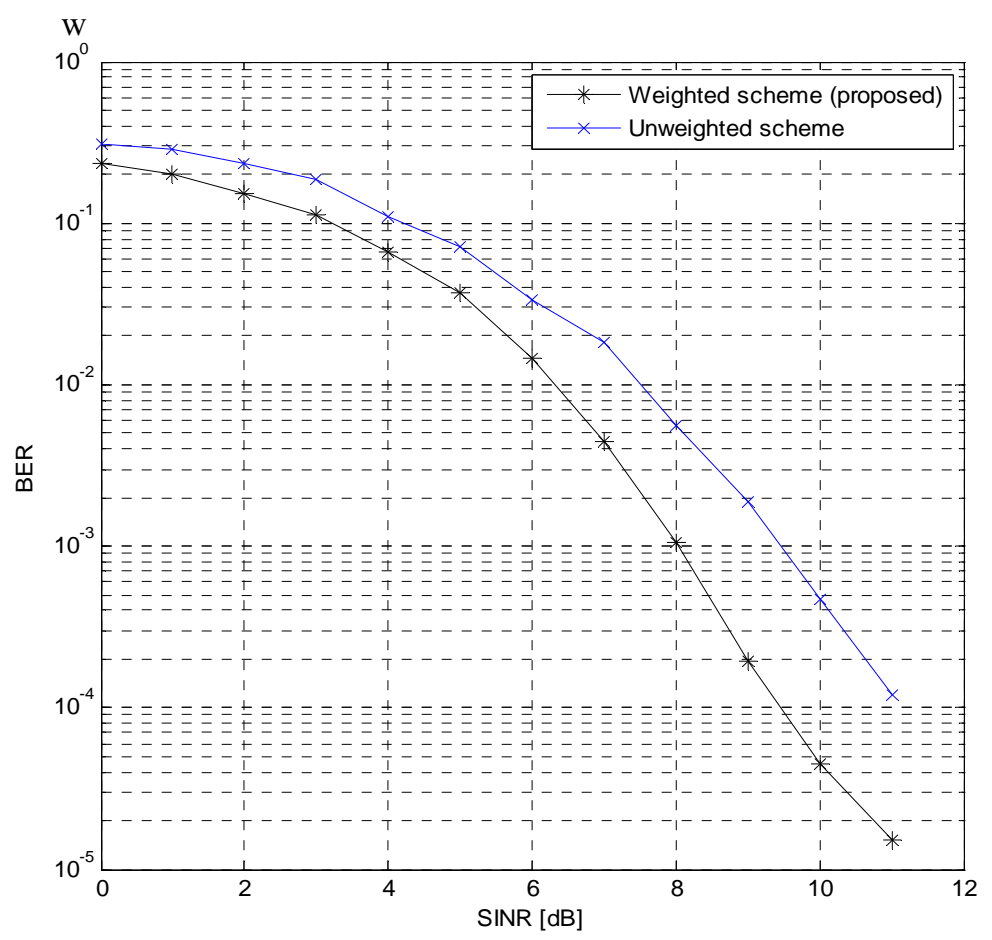

Figure 3. Bit error rate versus SINR.

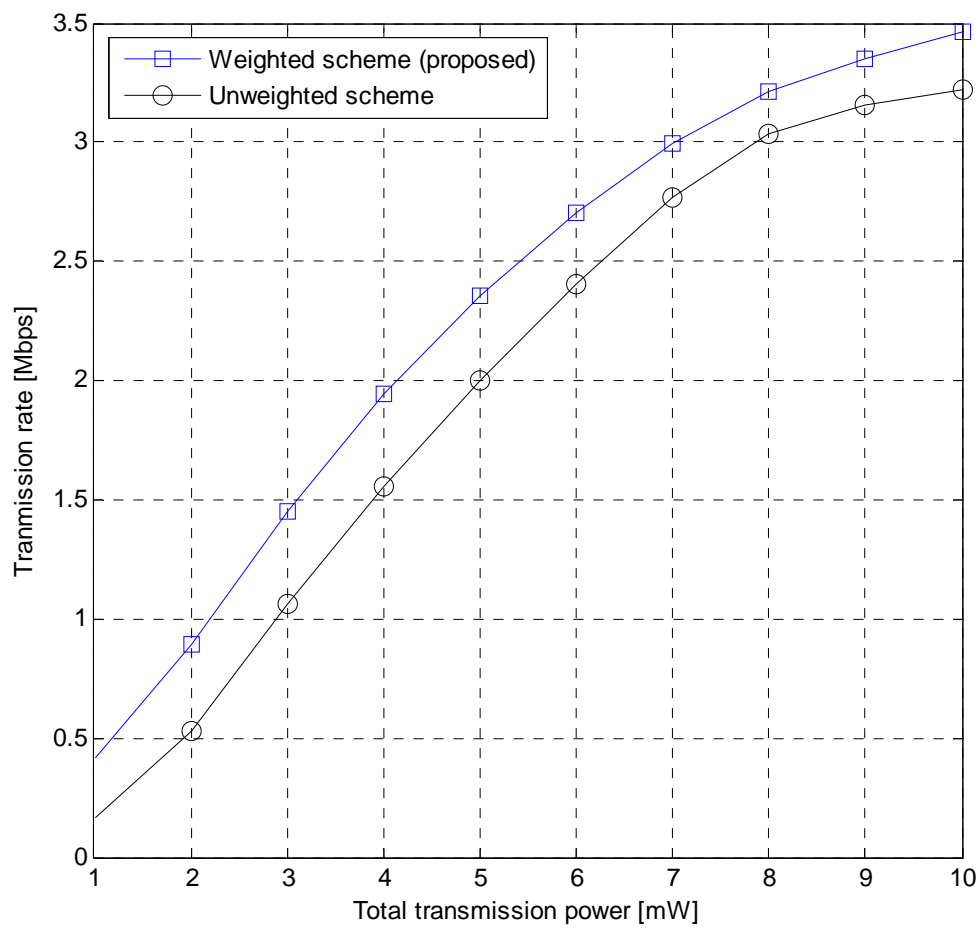

Figure 4. Transmission rate versus total transmission power in CR.

power. Here, the interference threshold is assumed to be $5 \times 10^{-4} \mathrm{~W}$. By increasing the total transmission power, transmission rate of the CR system increases. As can be seen from Figure 4, our proposed scheme achieves higher transmission rate than that of the other scheme. We ob- serve that at $5 \mathrm{~mW}$, our proposed scheme has $17 \%$ higher transmission rate compared with that of the scheme without applying power weights.

Figure 5 shows the transmission rate in terms of the interference introduced to the PUs by the CR system. In 


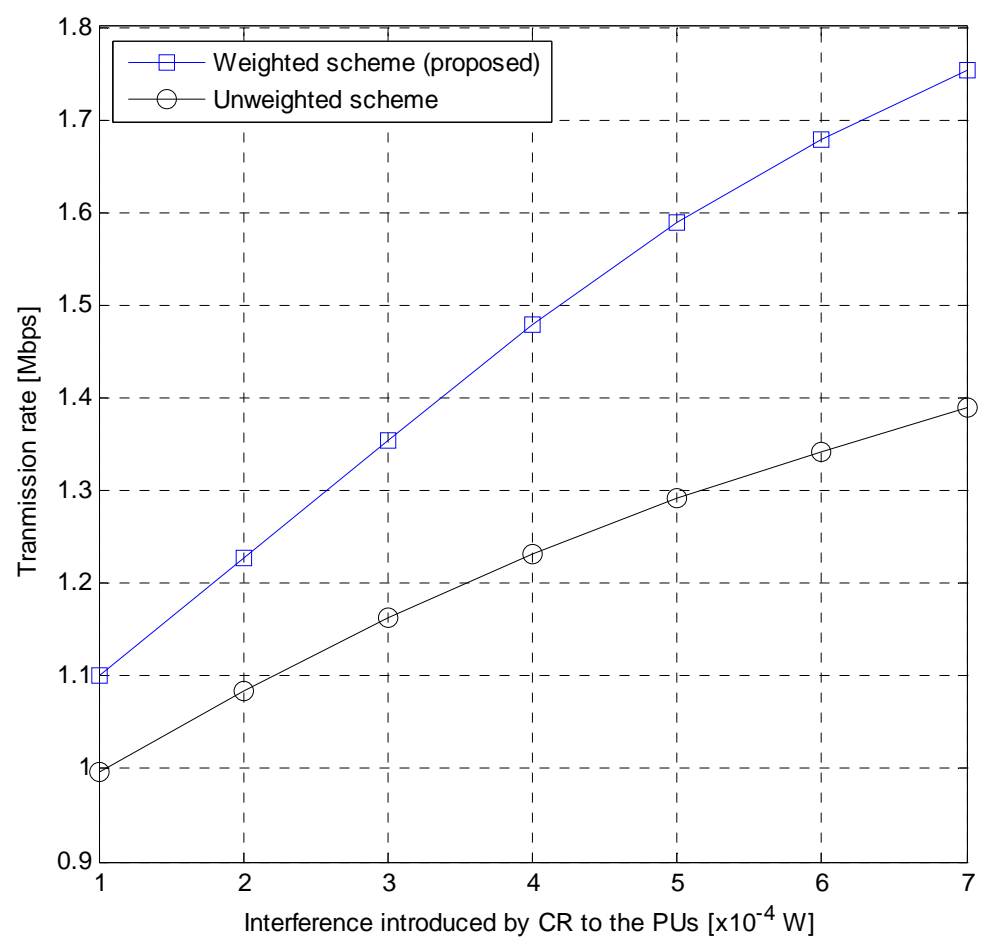

Figure 5. Transmission rate versus interference introduced by CR to the PUs.

this figure, we assume a fix total transmission power, which is equal to $3 \mathrm{~mW}$. By increasing the interference introduced to the PUs, transmission rate of the CR system increases. We observe that by increasing the interference introduced to the PUs, our proposed scheme provides a higher transmission rate compared with that of the other scheme. For example, at the interference power of $5 \times 10^{-4} \mathrm{~W}$, our proposed scheme achieves 1.6 Mbps while the same system without applying power weights achieves 1.3 Mbps. However, it's clear that the transmission rate of our proposed CR scheme in terms of interference power introduced to PUs is slower than that of the proposed scheme in terms of total transmission power (in previous figure).

\section{Conclusions}

In this paper, we have developed a new two phase scheme in cognitive radio systems based on LDPC-coded OFDM transmission. In the first phase, by maximizing total received $\mathrm{CR}$ signal powers in all $\mathrm{CR}$ subcarriers which provides higher transmission rate, adaptive power weights for CR subcarriers are achieved. This optimization problem has two constraints: 1) keeping the sum interference power introduced by CR to the PUs below a given threshold, 2) keeping the total transmit power of CR within a peak transmit power. In the second phase, by encountering unsteady noise plus interference gener- ated by PUs, we have applied LDPC decoder in the CR system. Moreover, by applying adaptive power weighted LLRs for CR subcarriers in each bit stream, error rate performance improves. Presented simulation results show that the proposed scheme achieves lower bit error rate and higher transmission rate compared with those of the same scheme without using adaptive power weights.

\section{Acknowledgements}

This work was partially funded by Iranian Institute of information and Communication Technology (the former ITRC). Also, the authors would like to thank Mr. Mohammad Bigdeli and Mr. Saeid Balaneshin from the wireless communication laboratory at IUST for their helpful comments and suggestions.

\section{References}

[1] S. Haykin, "Cognitive Radio: Brain-Empowered Wireless Communications," IEEE Journal on Selected Areas in Communications, Vol. 23, No. 2, 2005, pp. 201-220. doi:10.1109/JSAC.2004.839380

[2] T. Weiss and F. K. Jondral, "Spectrum Pooling: An Innovative Strategy for the Enhancement of Spectrum Efficiency," IEEE Communications Magazine, Vol. 43, No.3, 2004, pp. S8-S14. doi:10.1109/MCOM.2004.1273768

[3] G. Lechner, K. D. Nguyen, G. Fabregas and L. K. Rasmussen, "Optimal Power Control for LDPC Codes in Block- 
Fading Channels," IEEE Transactions on Communications, Vol. 59, No. 7, 2011, pp. 1759-1765. doi:10.1109/TCOMM.2011.051311.090238

[4] H. Futaki and T. Ohtsuki, "Performance of Low-Density Parity-Check (LDPC) Coded OFDM Systems," IEEE International Conference on Communications (ICC), Vol. 3, 2002, pp. 1696-1700. doi:10.1109/ICC.2002.997138

[5] M. Jiang, C. Zhao, E. Xu and L. Zhang, "ReliabilityBased Iterative Decoding of LDPC Codes Using Likelihood Accumulation," IEEE Communications Letters, Vol. 11, No. 8, 2007, pp. 677-679. doi:10.1109/LCOMM.2007.070450

[6] J. Lei, R. Yates, P. Spasojevic and L. Greenstein, "Cooperative Sensing of Primary Users in Cognitive Radio Networks Based on Message Passing," 43th Annual Conference on Information Sciences and Systems (CISS), Baltimore, 18-20 March 2009, pp. 568-573. doi:10.1109/CISS.2009.5054784

[7] A. Baker, S. Soumik Ghosh, A. Kumar and M. Bayoumi, "LDPC Decoder: A Cognitive Radio Perspective for Next Generation (XG) Communication," IEEE Circuits and Systems Magazine, Vol. 7, No. 3, 2007, pp. 24-37. doi:10.1109/MCAS.2007.904180

[8] B. Lu, Y. Guosen and W. Xiaodong, "Performance Analysis and Design Optimization of LDPC-coded MIMO OFDM Systems," IEEE Transactions on Signal Processing, Vol. 52 , No. 2, 2004, pp. 348-361.

[9] Y. Li and W. E. Ryan, "Mutual-Information-Based Adaptive Bit-Loading Algorithms for LDPC-Coded OFDM," IEEE Transactions on Wireless Communications, Vol. 6, No. 5, 2007, pp. 1670-1680. doi:10.1109/TWC.2007.360369
[10] A. Jovicic and P. Viswanath, "Cognitive Radio: An Information-Theoretic Perspective," IEEE Transactions on Information Theory, Vol. 55, No. 9, 2009, pp. 3945-3958.

[11] W. Lingfan, E. K. S. Au, P. W. C. Chan, R. D. Murch, R. S. Cheng, H. M. Wai and V. K. N. Lau, "Effect of Carrier Frequency Offset on Channel Estimation for SISO/MIMO OFDM Systems," IEEE Transactions on Wireless Communications. Vol. 6, No. 5, 2007, pp. 1854-1863. doi:10.1109/TWC.2007.360387

[12] S. E. Mahmoodi and B. Abolhassani, "Two New Power Allocation Schemes for an OFDM Cognitive Radio with No Knowledge on Primary Users' Interference," submitted to the International Journal of Communication Systems, 2011.

[13] T. Weiss, J. Hillenbrand, A. Krohn and F. K. Jondral, "Mutual Interference in OFDM-based Spectrum Pooling Systems," Proceeding of IEEE 59th Vehicular Technology Conference, Milan, 17-19 May 2004, pp. 1873-1877.

[14] S. Boyd and L. Vandenberghe, "Convex Optimization," Cambridge University Press, Cambridge, 2004.

[15] G. Bansal, M. J. Hossain and V. K. Bhargava, "Optimal and Suboptimal Power Allocation Algorithms for OFDMBased Cognitive Radio Systems," IEEE Transaction on Wireless Communications, Vol. 7, No. 11, 2008, pp. 47104718. doi:10.1109/T-WC.2008.07091

[16] S. Xiangran and Sh. Dongxin, "Design and Optimization of LDPC Encoder Based on LU Decomposition with Simulated Annealing," International Conference on Computer Science and Service System (CSSS), Nanjing, 27-29 June 2011, pp. 2181-2184. doi:10.1109/CSSS.2011.5974805 